\title{
CORRELAÇÕES ENTRE MORFOMETRIA OVARIANAE HORMÔNIOS EM VACAS ZEBUS NÃO PRENHES
}

\section{CORRELATIONS BETWEEN OVARIAN MORPHOMETRY AND HORMONES IN NO PREGNANT ZEBU COWS}

\author{
Chacur $^{1}$, M.G.M., E. Oba² e S.N. Kronka ${ }^{3}$
}

${ }^{1}$ Departamento de Reprodução Animal. Universidade do Oeste Paulista, UNOESTE. Rod. Raposo Tavares, km 572 s/n. Campus II. Presidente Prudente-SP. CEP 19067-175. Brasil. E-mail: chacur@unoeste.br ${ }^{2}$ Departamento de Reprodução Animal e Radiologia Veterinária. Universidade Estadual Paulista, UNESP. Botucatu, SP. Brasil.

${ }^{3}$ Departamento de Bioestatística. UNOESTE. Presidente Prudente-SP. Brasil.

\section{PalaVRas ChaVe ADICIONAIS}

Abatedouro. Folículo. Corpo lúteo. Progesterona. Cortisol.

\section{RESUMO}

O estudo da morfometria ovariana está diretamente ligado as suas aplicações práticas para realizar e interpretar os achados dos exames ginecológicos em vacas. O objetivo desse trabalho foi estudar a morfometria de ovários coletados em abatedouro e as correlações entre área do corpo lúteo, progesterona e cortisol em vacas zebu não prenhes. Foram coletados 114 pares de ovários em abatedouro, mensurados quanto a sua espessura, comprimento, largura e volume; diâmetro e volume do folículo, diâmetro e área do corpo lúteo, e concentrações de progesterona e cortisol. Foi observada diferença significativa $(p<0,05)$ para largura $1,95 \mathrm{~cm}$ e $1,83 \mathrm{~cm}$; e volume $(p<0,05) 7,26 \mathrm{ml}$ e $6,23 \mathrm{ml}$ do ovário esquerdo e direito, respectivamente. Houve correlação de $0,46(p<0,01)$ entre o volume do ovário esquerdo $x$ área do corpo lúteo. Na presença de folículos com diâmetro igual ou superior a $9 \mathrm{~mm}$, o corpo lúteo do tipo maciço e protruso presente em 23 $(43,39 \%)$ dos 53 ovários, predominou em relação ao tipo maciço e incluso 16 (30,18\%) dos ovários. Dos 84 ovários com corpos lúteos $26,20 \%$ eram do tipo incluso. Não houve correlação significativa entre área do corpo lúteo x progesterona, área do corpo lúteo $\mathrm{x}$ cortisol e progesterona $\mathrm{x}$ cortisol. Conclui-se que a presença de corpos lúteos inclusos, nas vacas zebu, pode resultar em falha durante o exame de palpação retal para estimar a atividade ovariana.

Recibido: 17-4-07. Aceptado: 5-11-07.

\section{ADDITIONAL KEYWORDS}

Slaughterhouse. Follicle. Corpus luteum. Progesterone. Cortisol.

\section{SUMMARY}

The study of ovarian morphometry is directly related to its practical applications to interpret the examination of the reproductive tract of the cow. The objective of this work was to study the ovarian morphometry collected in slaughterhouse and correlations between corpus luteum area, progesterone and cortisol in no pregnant zebu cows. One hundred fourteen pairs of ovaries were collected from slaughterhouse, measured in thickness, length, width and volume, diameter and volume of the follicle, diameter and area of the corpus luteum. Significative difference $(p<0.05)$ were observed for widths of $(1.95 \mathrm{~cm}$ and 1.83 $\mathrm{cm})$ and volume $(p<0.05)$ of $7.26 \mathrm{ml}$ and $6.23 \mathrm{ml}$ of left and right ovaries respectively. For size and volume of follicles, and diameter and area of the corpus luteum, there was no significative difference between the sides. There was correlation of $0.46(p<0.01)$ between left ovary volume $\mathrm{x}$ luteum corpus area, in the presence of follicles with diameters equal or greater than $9 \mathrm{~mm}$, the corpus luteum of the massive and protuberant type were present in 23 (43.39\%) of 53 ovaries, predominating in relation to type of massive and incluse $16(30.18 \%)$. There were no significative correlation between corpus luteum area $\mathrm{x}$ progesterone, corpus luteum area $\mathrm{x}$ cortisol and progesterone $x$ cortisol. In conclusion, the presence of the included corpus luteum in zebu cows could result in error during the rectal palpation

Arch. Zootec. 58 (223): 467-470. 2009. 


\section{CHACUR, OBAE KRONKA}

exam to estimate the ovarian activity.

\section{INTRODUÇÃO}

$\mathrm{Na}$ formação do corpo lúteo $(\mathrm{CL})$ na espécie bovina, o tecido pode proliferar ocupando apenas a área interior do ovário, o que resulta em CL incluso ou extrapolando para fora do ovário formando um ápice na superfície, classificado como CL protruso (Neves et al., 2002).

A presença de cavidade pode ser observada no centro de alguns corpos lúteos, tendo maior incidência no CL em desenvolvimento e menor no já desenvolvido (Okuda et al., 1988). Desta forma, objetivouse estudar a morfometria de ovários coletados em abatedouro e as correlações entre área do corpo lúteo, progesterona e cortisol em vacas zebu não prenhes.

\section{MATERIAL E MÉTODOS}

Foi realizado um estudo por meio de coleta de 114 pares de ovários, de vacas zebus não prenhes, recuperados em abatedouro no município de Presidente Prudente, SP. Anteriormente ao abate e à obtenção dos ovários, colheitas de sangue foram efetuadas para as dosagens de progesterona e cortisol, obtidas da veia jugular das vacas em diestro, identificadas por meio de palpação retal, perante a presença de CL, realizada entre os dias 7 e 14 após o estro, sendo o cio identificado por observação prévia individual dos animais. As amostras de sangue foram imediatamente centrifugadas, durante 15 minutos a 336,3 g, para a separação do soro, armazenado em criotubos, identificados e estocados a $-20^{\circ} \mathrm{C}$, até seu processamento. As análises de progesterona e cortisol foram realizadas por radioimunoensaio (RIA).

Os ovários foram coletados imediatamente após o abate e evisceração dos animais, identificados quanto ao lado em ovário direito (OD) e esquerdo (OE); armazenados e transportados em solução salina a $37^{\circ} \mathrm{C}$.
Mensurou-se os ovários com paquímetro quanto ao comprimento $(\mathrm{cm})$ no eixo maior, largura $(\mathrm{cm})$ no eixo entre o pedículo do ovário e a extremidade oposta; e espessura (cm) no eixo de $90^{\circ}$ em relação ao eixo da largura. O volume $(\mathrm{ml})$ dos ovários foi obtido por meio da imersão individual dos mesmos em proveta graduada, contendo solução fisiológica.

O maior folículo presente nos ovários direito e esquerdo foi identificado e mensurado quanto ao seu diâmetro $(\mathrm{mm})$ e volume (ml), calculado pela equação do volume da esfera. A área de tecido luteal foi obtida a partir da área da elipse, definida pelo maior e menor diâmetros do corpo lúteo (CL), sendo classificados em corpo lúteo 1 (CL 1): área até $1 \mathrm{~cm}^{2},(\mathrm{CL} 2)$ : área de 1,1 a 2 $\mathrm{cm}^{2}$, (CL 3): área de 2, 1 a $3 \mathrm{~cm}^{2}$ e (CL 4): área igual ou superior a $3,1 \mathrm{~cm}^{2}$. Morfologicamente, os corpos lúteos foram denominados de: CL incluso, quando da localização total do tecido luteal abaixo da superfície do ovário; ou CL protruso, apresentando uma porção luteal acima da superfície do ovário, segundo Neves et al. (2002). Anotou-se a presença de cavidade nos corpos lúteos (CL cavitário), ou ausência da cavidade (CL maciço).

Os resultados das médias foram submetidos à análise de variância pelo teste $\mathrm{F}$, de acordo com um delineamento inteiramente casualizado, segundo Banzatto e Kronka (2006), cujo modelo matemático é:

$$
\mathrm{x}_{\mathrm{ij}}=\mathrm{m}+\mathrm{t}_{\mathrm{i}}+\mathrm{e}_{\mathrm{ij}}
$$

em que:

$\mathrm{x}_{\mathrm{ij}}=$ valor observado na parcela do lado ${ }_{\mathrm{i}}$ e repetição ${ }_{\mathrm{j}}$ $\mathrm{m}=$ média geral

$t_{i}=$ efeito do lado

$\mathrm{e}_{\mathrm{ij}}=$ efeito do acaso.

Para alguns pares de medições de interesse foi calculado o coeficiente de correlação linear, cujo teste foi o t de Student. Para o número de corpos lúteos foi aplicado o teste de qui-quadrado $\left(\chi^{2}\right)$. 


\section{MORFOMETRIA OVARIANAE HORMÔNIOS EM VACAS ZEBUS NÃO PRENHES}

\section{RESULTADOSEDISCUSSÃO}

Para a largura e volume dos ovários, houve diferença $(p<0,05)$ entre o lado esquerdo e direito com $1,95 \mathrm{~cm}$ e $1,83 \mathrm{~cm}$; e de 7,26 ml e 6,23 ml, respectivamente, sendo superior aos relatos de Neves et al.(2002) para o volume ovariano do lado esquerdo $(6,37 \mathrm{ml})$ e inferior ao direito $(7,16 \mathrm{ml})$. $\mathrm{Na}$ largura, os valores do presente estudo foram superiores ao de $1,61 \mathrm{~cm}$ para o ovário esquerdo e de $1,71 \mathrm{~cm}$ para o direito (Neves etal.,2002).

Sendo as médias do presente estudo superior às encontradas para vacas zebus, com 2,57 cm de comprimento para o ovário esquerdo e 2,62 cm para o direito; e também superior em relação ao valor de $1,22 \mathrm{~cm}$ de espessura no ovário esquerdo e $1,21 \mathrm{~cm}$ no direito, relatado por Neves et al.(2002).

Os maiores coeficientes de variação foram encontrados para o volume folicular $(\mathrm{CV}=143,14 \%)$ e área do $\mathrm{CL}(\mathrm{CV}=49,33 \%)$.

Houve correlação de $0,46(p<0,01)$ entre o VO e a área do CL. No presente estudo, essa diferença pode estar associada aos $65,47 \%$ de corpos lúteos presentes no lado esquerdo, sendo que $73,80 \%$ deles eram protrusos, levando ao aumento significativo das dimensões ovarianas (tabela I).

A média do tamanho dos maiores folículos $8,22 \pm 3,99 \mathrm{~mm}$ influenciou de forma

Tabela I. Número e percentagem dos tipos de corpos lúteos (CL) de vacas zebus não prenhes. (Number and percentage of corpus luteum types in non-pregnant zebu cows).

\begin{tabular}{lcc}
\hline Característica do ovário & $\mathrm{n}$ & $(\%)$ \\
\hline CL ovário esquerdo & 55 & 65,47 \\
CL ovário direito & 29 & 34,53 \\
Total de ovários com CL & 84 & 100 \\
CL cavitário & 15 & 17,85 \\
CL maciço & 69 & 82,14 \\
CL incluso & 22 & 26,19 \\
CL protruso & 62 & 73,80 \\
Total de ovários com CL & 84 & \\
& & \\
\hline
\end{tabular}

negativa no volume do ovário direito 6,23 $\mathrm{ml}$. Essa observação pode parecer contraditória, uma vez que a presença de um folículo de maior tamanho deveria resultar em um ovário com dimensões superiores. O folículo dominante determina a atresia dos folículos subordinados e inibe o recrutamento de novos folículos (Ginther, 1997).

Existem diferenças funcionais entre os corpos lúteos maciços e cavitários, mas a presença da cavidade não está relacionada com condições patológicas (Okuda et al., 1988). Corpos lúteos que apresentam cavidade podem ser encontrados em até $86 \%$ das fêmeas bovinas gestantes e $77 \%$ das não gestantes, sendo sua provável origem atribuída à ocupação incompleta da cavidade folicular pelas células durante a luteinização (Kastelic et al., 1990). Os corpos lúteos foram classificados como cavitários quando o diâmetro de suas cavitações eram superiores a $1 \mathrm{~cm}$ (McEntee, 1990), estando o tamanho da cavidade relacionado com o estágio de desenvolvimento do tecido luteal, sendo a mesma maior na fase de formação do $\mathrm{CL}$ em relação ao $\mathrm{CL}$ desenvolvido (Okuda et al., 1988).

Os ovários com CL cavitário estiveram presentes em $17,85 \%$ em relação ao total de corpos lúteos presentes (tabela I), similar aos $16,3 \%$ relatado por Neves et al. (2002) em vacas zebu. Houve predomínio de corpos lúteos maciços com 82,14\% (tabela I), incidência essa semelhante à descrita por Okuda et al. (1988) de 83,7\%.

No presente estudo, não houve diferença significativa entre o lado direito e esquerdo; na presença de folículos com diâmetros iguais ou superiores a $9 \mathrm{~mm}$, nos ovários com corpos lúteos cavitários ou maciços. Essa observação, provavelmente, está associada ao fato de que folículos são identificados no transcorrer do ciclo estral, vindo a sofrer atresia devido à produção de progesterona pelo CL, concordando com as observações para ovários obtidos de vacas da raça Holandesa, recuperados em matadouro (Okuda et al., 1988). 


\section{CHACUR, OBAE KRONKA}

Tabela II. Correlações entre: área do corpo lúteo, CL 1: até $1 \mathrm{~cm}^{2}$; CL 2: 1,1 a $2 \mathrm{~cm}^{2}$; CL 3: 2,1 a $3 \mathrm{~cm}^{2}$ e CL 4: igual ou superior a 3,1 $\mathrm{cm}^{2}$, progesterona, $\mathrm{P}_{4}$ e cortisol, $\mathrm{C}$ de vacas zebus não prenhes. (Correlations between corpus luteum area, $\mathrm{CL}$ 1: untill $1 \mathrm{~cm}^{2} ; \mathrm{CL} 2: 1,1$ to 2 $\mathrm{cm}^{2}$; CL 3: 2,1 to $3 \mathrm{~cm}^{2} \mathrm{e} C L$ 4: equal or superior to $3,1 \mathrm{~cm}^{2}$, progesterone, $\mathrm{P}_{4}$ e cortisol, $\mathrm{C}$ of no pregnant zebu cows).

\begin{tabular}{lcccc}
\hline Correlações & CL 1 & CL 2 & CL 3 & CL 4 \\
\hline Área CL x P & $-0,34^{\mathrm{NS}}$ & $0,19^{\mathrm{NS}}$ & $-0,41^{\mathrm{NS}}$ & $-0,14^{\mathrm{NS}}$ \\
Área CL x C & $0,25^{\mathrm{NS}}$ & $0,17^{\mathrm{NS}}$ & $-0,08^{\mathrm{NS}}$ & $0,09^{\mathrm{NS}}$ \\
$\mathrm{P}_{4} \times \mathrm{C}$ & $-0,04^{\mathrm{NS}}$ & $0,20^{\mathrm{NS}}$ & $0,25^{\mathrm{NS}}$ & $0,25^{\mathrm{NS}}$ \\
\hline
\end{tabular}

NS= não significativo a $5 \%$.

Para o número e porcentagem de corpos lúteos, presentes em 84 (36,84\%) ovários de um total de 228 observou-se $55(65,47 \%)$ no lado esquerdo e $29(34,53 \%)$ no lado direito. Dos 84 ovários com CL, 22 (26,19\%) eram do tipo incluso e $62(73,80 \%)$ do tipo protruso (tabela I), sendo esse um achado de utilização prática, uma vez que a estrutura luteinizada, inclusa no ovário, pode não ser detectada

\section{BIBLIOGRAFIA}

Banzatto, D.A. e S.N. Kronka. 2006. Experimentação agrícola. $4^{a}$ ed. FUNEP. Jaboticabal. $237 \mathrm{p}$.

Ginther, O.J. 1997. Emergency and deviation of follicles during the development of follicular waves in cattle. Theriogenology, 48: 75-87.

Hasler, J.F., R.A. Bowen and L.D. Nelson. 1980. Serum progesterone concentrations in cows receiving embryo transfer. J. Reprod. Fertil., 58: 71-77

Kastelic, J.P., R.A. Pierson and O.J. Ginther. 1990. Ultrasonic morphology of corpora lutea and central cavities during the estrour cycle and early pregnancy in heifers. Theriogenology, 33: 487-498.

McEntee, K. 1990. Reproductive pathology of domestic animals. Academic Press. San por meio da palpação retal, durante a avaliação ginecológica, podendo levar ao diagnóstico equivocado quanto à atividade ovariana luteal cíclica, concordando com Radostitis e Blood (1986).

Não houve correlações significativas entre área do corpo lúteo x progesterona, área do corpo lúteo x cortisol e progesterona $\mathrm{x}$ cortisol (tabela II), semelhante ao relatado por Hasler et al. (1980). Sugere-se que o dia do ciclo e a raça possam influenciar na correlação entre área do CL e produção de $\mathrm{P}_{4}$. Ribadu et al. (1994) compararam a avaliação de corpos lúteos de meio de ciclo, por meio de palpação retal e níveis plasmáticos de progesterona, relatando sensitividade de $85 \%$ e predictibilidade positiva de $89,7 \%$, respectivamente.

Em conclusão, a presença de corpos lúteos do tipo incluso nas vacas zebu sugere que a palpação retal das estruturas ovarianas nem sempre é eficaz para predizer a atividade ovariana luteal cíclica. A produção de progesterona e de cortisol não revelou correlação com a área do corpo lúteo, sendo esse um achado importante para a avaliação ginecológica em vacas zebu.

Diego. 425 p.

Neves, M.M., A.P. Marques Júnior, C.V. Santana, F.P.C. Lima e W.J. Zambrano. 2002. Características de ovários de fêmeas zebu (Bos taurus indicus), colhidos em abatedouros. Arq. Bras. Med. Vet. Zoo., 54: 1-5.

Okuda, K., S. Kito and K. Sato. 1988. A Study of the central cavity in the bovine corpus luteum. Vet. Rec., 123: 180-183.

Radostitis, O.M. e D.C. Blood. 1986. Manual de controle da saúde e produção de animais. $2^{a}$ ed. Manole. São Paulo. 189 p.

Ribadu, A.Y., W.R. Ward and H. Dobson. 1994. Comparative evaluation of ovarian structures in cattle by palpation per rectum, ultrasonography and plasma progesterone concentration. Vet. Rec., 135: 452-457. 